potentials, proposed in the present work, is based on the extrapolation of an approximately linear pattern obtained in horizontal analysis whose linearity is easily justified on the basis of the linear theory. ${ }^{7}$

(c) Only the relative electron affinities obtained here are significant, because the lack of sufficiently precise ionization potentials in the fourth and higher spectra $(q=3)$ do not permit an accurate absolute determination.

(d) The deviations from the linear pattern are significant, but do not change the results qualitatively or in order of magnitude.

(e) The final computed affinities, after adjustment with $\mathrm{O}^{-}(1.465 \pm 0.005)^{10}$ and $\mathrm{Cl}^{-}(3.69 \pm 0.05)^{11}$ agree

${ }^{10}$ L. M. Branscomb, D. S. Burch, S. J. Smith, and S. Geltman, Phys. Rev. 111, 504 (1958).

${ }_{11}$ D. Cubicciotti, J. Chem. Phys. 31, 1646 (1959). within experimental error with the measurements on $\mathrm{F}^{-}(3.48 \pm 0.05)^{11}$ and $\mathrm{S}^{-}(2.07 \pm 0.07),{ }^{12}$ but are about $10 \%$ higher than the recent value of $\mathrm{C}^{-}(1.25 \pm 0.03) .^{13}$ No accurate measurements on the other computed negative atomic ions seem to exist.

(f) The results of the method proposed here can be improved in two respects: The slopes and intercepts can be extrapolated better when more accurate ionization potentials become available, and the values for the configuration centers $C\left(-1, p^{n}\right)$ can be improved by going beyond the quadratic extrapolation of the excited states.

${ }^{12}$ L. M. Branscomb and S. J. Smith, J. Chem. Phys. 25, 598 (1956).

${ }^{13}$ L. M. Branscomb and M. Seman, 2nd Intnl. Conf. on Electronic and Atomic Collisions, University of Colorado, 1961.

\title{
Motion of Small Suspended Particles in Nonuniform Gases*
}

\author{
EDWARD A. Mason \\ Institute for Molecular Physics, University of Maryland, College Park, Maryland \\ AND \\ Sydney Chapman \\ High Altitude Observatory, Boulder, Colorado; $\dagger$ Geophysical Institute, College, Alaska; \\ Institute of Science and Technology, University of Michigan, Ann Arbor, Michigan
}

(Received July 26, 1961)

\begin{abstract}
The motion of small suspended particles in a gas or gas mixture containing gradients of temperature, pressure, or composition is derived as a special case of the Chapman-Enskog kinetic theory of gases, by formally treating the suspended particles as large molecules. Gas molecules colliding with the suspended particles are considered to rebound elastically, but a fraction $f$ rebound in random directions and the remainder rebound specularly. The results check, in an indirect way, the calculations of Waldmann by a momentum transfer method on a slightly different model, in which the randomly rebounding molecules also have a random distribution of speeds. Significantly different results are predicted by the two models only in the presence of a temperature gradient (thermal diffusion), which has interesting implications concerning thermal diffusion in polyatomic gases.
\end{abstract}

\section{INTRODUCTION}

$\mathbf{T}$ HE motion of small suspended particles (dust, smoke, aerosols, etc.) in a gas or gas mixture containing gradients of temperature, pressure, or composition, is a subject which enters into the discussion of a great variety of phenomena of interest in such diverse fields as astrophysics, geophysics, colloid chemistry, and chemical engineering. The subject goes back at least to 1870 , when Tyndall ${ }^{1}$ noticed a dust-free

\footnotetext{
* Supported in part by the U. S. Atomic Energy Commission. $\dagger$ There engaged on a program of research sponsored by the National Bureau of Standards and the Air Force Geophysical Research Directorate.

1 J. Tyndall, Proc. Roy. Inst. 6, 3 (1870). For a review of this and other early work see P. Rosenblatt and V. K. LaMer, Phys. Rev. 70, 385 (1946).
}

region in the gas space about a hot body, showing that a temperature gradient has an effect on the motion of dust particles in a gas. Not surprisingly, there is a large body of literature, both experimental and theoretical, connected with the subject. Recently, Wald$\operatorname{mann}^{2}$ has given a very complete theory, based on a model which is somewhat simplified, but which is physically realistic enough to furnish a sound basis for the discussion of many phenomena. The model is that of spherical particles (smaller than the mean free path of the gas molecules) which in collisions with gas molecules reflect a fraction $a$ of the gas molecules

${ }^{2}$ L. Waldmann, Z. Naturforsch. 14a, 589 (1959). For a review of some earlier work using the same calculation method, see S. P. Bakanov and B. V. Derjaguin, Discussions Faraday Soc. 30, 130 (1960). 
diffusely with a Maxwellian distribution of speeds, and reflect the remaining fraction 1- $a$ specularly with no change in speed.

Waldmann directly calculates the force on a particle by adding up all the impulses transferred to it by the colliding gas molecules, using the known ChapmanEnskog velocity distribution for a gas which is not in equilibrium. From the force it is straightforward to compute the average drift velocity or the flux of the particles. Waldmann's results have been verified experimentally for aerosol droplets in a gas with a temperature gradient ${ }^{3}$ and in a diffusing gas mixture. ${ }^{4}$

The purpose of the present paper is to derive and slightly extend Waldmann's results as special cases of the Chapman-Enskog theory of gases, insofar as this is possible, by considering the suspended particles as large molecules. The new feature introduced by Waldmann is the fraction $a$ of "thermally diffuse" collisions; except for this, many of his results are already known under the special case of a so-called quasi-Lorentzian gas, ${ }^{5}$ as Waldmann pointed out. Since the thermally diffuse model involves inelastic collisions, which are not handled by the ChapmanEnskog theory, it is necessary to modify the model somewhat. Our results are therefore not identical with Waldmann's, but they are very similar in both algebraic form and numerical magnitude, with one interesting exception. This is the case of the motion of particles due to a temperature gradient in the gas, where the results depend on whether or not inelastic collisions occur. This has implications for the problem of thermal diffusion in polyatomic gases.

\section{MODEL}

We choose a model similar to Waldmann's, but one which preserves elastic collisions. This model also assumes spherical particles (smaller than the molecular mean free path in the gas), which reflect a fraction $f$ of the gas molecules diffusely and a fraction 1- $f$ specularly; but the diffusely reflected molecules are reflected elastically (i.e., with unchanged relative speed) instead of with a random Maxwellian distribution. In this "elastically diffuse" collision model, the directions but not the speeds of the molecules can be "accommodated" on collision with the large particle. This model is then analogous to the case where colliding molecules can follow different force laws, depending on the electron spin orientation on collision. The simplest example is the collision of two hydrogen atoms, in which $\frac{1}{4}$ of the collisions follow the force law corresponding to the ${ }^{1} \Sigma$ ground state of $\mathrm{H}_{2}$ (electron spins paired), and the other $\frac{3}{4}$ follow the force law corresponding to the ${ }^{3} \Sigma$ repulsive state of $\mathrm{H}_{2}$ (electron spins parallel). The fraction 1-f thus corresponds to a "ground state," and

\footnotetext{
K. H. Schmitt, Z. Naturforsch. 14a, 870 (1959).

${ }^{4} \mathrm{~K}$. H. Schmitt and L. Waldmann, Z. Naturforsch. $15 a, 844$ (1960)
}

E. A. Mason, J. Chem. Phys. 27, 782 (1957). the fraction $f$ to a collection of excited states, each with a probability of occurrence proportional to the cosine of the angle between the path of the reflected molecule and the normal to the particle surface (according to the usual cosine law of diffuse reflection ${ }^{6}$ ). In such a situation the results of the Chapman-Enskog theory remain formally the same, but the collision integrals are calculated slightly differently, being weighted averages over all the possible force laws. ${ }^{7}$

To carry out this averaging for diffuse reflection, in which the weighting factor involves the deflection angle, it is better to drop the usual classical impact parameter formulation and go over to the differential scattering cross section formulation, just as in the quantum theory of transport phenomena. ${ }^{8}$ The analogy to the quantum treatment is really quite close, since in both cases the angle of deflection is not uniquely determined by the initial relative speed and the impact parameter. We thus make the replacement

$$
b d b \rightarrow \sigma(\chi) \sin \chi d \chi,
$$

where $b$ is the impact parameter, $\sigma(\chi)$ is the differential scattering cross section, and $\chi$ is the deflection angle. Then, according to the model,

$$
\sigma(\chi)=(1-f) \sigma(\text { specular })+f \sigma(\text { diffuse }) .
$$

For spherical particles of radius $r$ impinged upon by molecules of size negligible compared to $r$, it is well known that ${ }^{9}$

$$
\sigma(\text { specular })=\frac{1}{4} r^{2} .
$$

The calculation of $\sigma$ (diffuse) is a straightforward problem in geometry, but it is a little involved and is therefore put in the Appendix. The result is

$$
\begin{aligned}
& \sigma(\text { diffuse })=\left(\frac{1}{16} r^{2}\right)(\sin \chi)^{-1}(\sin 2 \chi-2 \chi \cos 2 \chi), \\
& \text { for } 0 \leq \chi \leq \frac{1}{2} \pi ; \\
& \sigma(\text { diffuse }) \\
& \quad=\left(\frac{1}{16} r^{2}\right)(\sin \chi)^{-1}[-3 \sin 2 \chi+2(3 \chi-2 \pi) \cos 2 \chi], \\
& \text { for } \frac{1}{2} \pi \leq \chi \leq \pi
\end{aligned}
$$

This checks in giving a value of $\pi r^{2}$ for the total scattering cross section,

$$
Q=2 \pi \int_{0}^{\pi} \sigma(\chi) \sin \chi d \chi
$$

as it should.

\footnotetext{
- R. D. Present, Kinetic Theory of Gases (McGraw-Hill Book Company, Inc., New York, 1958), pp. 56-57.

${ }^{7}$ E. A. Mason, J. T. Vanderslice, and J. M. Yos, Phys. Fluids 2, 688 (1959).

${ }^{8} \mathrm{~S}$. Chapman and T. G. Cowling, The Mathematical Theory of Non-Uniform Gases (Cambridge University Press, New York, 1952), Chap. 17.

Reference 6, pp. 140-141.
} 
For the reduced diffusion cross section, defined $a^{10}$

$$
Q^{(1) *}=\frac{2 \pi}{\pi r^{2}} \int_{0}^{\pi}(1-\cos \chi) \sigma(\chi) \sin \chi d \chi
$$

we obtain from Eqs. (3) and (4) the results

$$
Q^{(1) *}(\text { specular })=1, Q^{(1) *}(\text { diffuse })=\frac{13}{9} \text {. }
$$

The first result follows from the normalization factor used in the definition, and the second result checks that obtained by Epstein ${ }^{11}$ for the same model, using momentum transfer methods such as Waldmann used. Carrying the procedure through to the average reduced collision integral ${ }^{10}$ for diffusion, we obtain

$$
\left\langle\Omega^{(1,1) *}\right\rangle=(1-f)(1)+(f)\left(\frac{13}{8}\right)=1+\frac{4}{8} f .
$$

For the thermally diffuse scattering model, Waldmann obtains a similar result with $(\pi / 8) a$ in place of $\frac{4}{8} f$. The maximum difference between the models is thus only $3.7 \%$ (when $f$ and $a$ are both unity), at least as far as diffusion is concerned.

It should be remarked that the foregoing calculations assume that there is no interaction between the large particles and a gas molecule until they are in contact. This restriction is probably satisfactory if $r$ is large enough, but could easily be relaxed to allow for a weak interaction at greater separations than $r$ by the wellknown Sutherland approximation. ${ }^{12}$ The effect of this is to increase the collision integral by the factor $[1+(S / T)]$, where $S$ is the Sutherland constant and $T$ is the absolute temperature. This refinement is probably seldom, if ever, needed, and we do not make it here.

\section{RESULTS}

Results analogous to Waldmann's are now easy to obtain as special cases of the Chapman-Enskog kinetic theory. We first calculate the transport coefficients, from these the average velocity or flux of the suspended particles, and finally the average force on a particle. This procedure is just the reverse of Waldmann's. We assume, with Waldmann, that the mole fraction of suspended particles is very small, and that the mass of a particle is much greater than the mass of a gas molecule. This latter condition means that the reduced mass of a colliding molecule-particle pair is equal to the mass of the molecule alone. These conditions considerably simplify the mathematics of the ChapmanEnskog theory, and define what has been called a quasi-Lorentzian gas. ${ }^{5}$ The binary diffusion coefficient for such a gas is

$$
\frac{1}{D_{12}}=\frac{8 n}{3}\left(\frac{2 m_{2}}{\pi k T}\right)^{3}\left(\pi r^{2}\right)\left(1+\frac{4}{9} f_{2}\right),
$$

\footnotetext{
${ }^{10} \mathrm{~J}$. O. Hirschfelder, C. F. Curtiss, and R. B. Bird, Molecular Theory of Gases and Liquids (John Wiley \& Sons, Inc., New York, 1954), Chap. 8.

11 P. S. Epstein, Phys. Rev. 23, 710 (1924).

12 Reference 8, Chap. 10.
}

where $n=p / k T$ is the total molecular density. This expression is exact for the model, not just a first approximation. ${ }^{5}$ We conform to the convention that the subscript 1 refers to the heavy component and the subscript 2 to the light component (this distinction is unimportant except for the case of thermal diffusion, where it determines the sign convention for the separation). Thus the subscript 1 always refers to the suspended particles. Equation (8) corresponds to Waldmann's Eq. (5.5), but has $\frac{4}{8} f$ in place of $\left(\frac{1}{8} \pi\right) a$. The only other expressions we need are the collision integral ratios ${ }^{10} B_{12}{ }^{*}$ and $C_{12}{ }^{*}$, which are readily found to be unity for rigid spheres, whether reflection is diffuse or specular. From these expressions we can write down the results for the following particular cases.

\section{A. Frictional Force in a Flowing Gas}

The easiest way to compute the force on a stationary suspended particle due to a flowing gas (or homogeneous gas mixture) is to imagine the gas stationary and the particle moving through it under the influence of an external force. This is then just the kinetic theory case of forced diffusion. For concreteness, we can imagine putting a small electrical charge on the particle and forcing it through the gas by an electric field. In this case we can simply take over the kinetic theory of ion mobility, which yields the following exact expression for a quasi-Lorentzian gas ${ }^{13}$ :

$$
\bar{v}_{1}=K_{1} F_{1},
$$

where $\bar{v}_{1}$ is the average (drift) velocity of the particles, $F_{1}$ is the force on the particles, and $K_{1}$ is the mobility. For a pure gas, $K_{1}=D_{12} / k T$, and the force is therefore

$$
F_{1}=k T \bar{v}_{1} / D_{12}=\frac{8}{8} r^{2} n\left(2 \pi m_{2} k T\right)^{\frac{1}{2}}\left(1+\frac{4}{8} f_{2}\right) \bar{v}_{1},
$$

which corresponds to Waldmann's Eq. (3.8) except that $\frac{4}{9} f$ replaces $\left(\frac{1}{8} \pi\right) a$. If the particle is moving at a steady velocity through the gas (i.e., is not accelerated), then the gas must exert an equal and opposite frictional force $-F_{1}$ on the particle. If the particle is considered stationary, then $F_{1}$ is the force on it caused by the gas flowing by with velocity $\bar{v}_{1}$.

These results are easily extended by the present method to flowing gas mixtures. The reciprocal of the mobility in the mixture is just the sum of the reciprocals of the mobilities in all the pure components, weighted by their mole fractions in the mixture. (This is usually known as Blanc's law in ion mobility work.) For a flowing gas composed of a mixture of species $2,3, \cdots, \nu$, Eq. (10) becomes

$$
F_{1}=\frac{8}{3} r^{2} n(2 \pi k T)^{\frac{1}{2}} \bar{v}_{1} \sum_{i=2}^{p} x_{i} m_{i}^{1}\left(1+\frac{4}{9} f_{i}\right)
$$

where $x_{i}$ is the mole fraction of species $i$.

${ }^{18} \mathrm{~T}$. Kihara, Revs. Modern Phys. 25, 844 (1953). See also E. A. Mason and H. W. Schamp, Jr., Ann. Phys. (N. Y.) 4, 233 (1958). 


\section{B. Motion in a Pressure Gradient}

This case was not considered by Waldmann, but may be of some interest in problems in atmospheric physics, where pressure gradients are caused by the gravitational field. ${ }^{10}$ In general, for any binary gas mixture at constant temperature, the flux equation for one component can be written as follows in a spacefixed (laboratory) coordinate system ${ }^{10}$ :

$$
J_{1}=n_{1} \bar{v}_{1}=-n D_{12} \nabla x_{1}+\frac{n^{2} x_{1} x_{2}\left(m_{1}-m_{2}\right)}{\rho} D_{12} \nabla \ln p+n_{1} u,
$$

where $J_{1}$ is the 1 -flux in molecules/ $\mathrm{cm}^{2}$-sec, $n_{1}$ is the 1 -number density in molecules $/ \mathrm{cm}^{3}, \rho=n_{1} m_{1}+n_{2} m_{2}$ is the total density in $\mathrm{g} / \mathrm{cm}^{3}, x_{1}$ and $x_{2}$ are the mole fractions, and $u$ is the number-average velocity,

$$
u=(1 / n)\left(n_{1} \bar{v}_{1}+n_{2} \bar{v}_{2}\right) .
$$

The first term of (12) gives the transport due to concentration diffusion, the second term that due to pressure diffusion, and the third term that due to any net flow.

If the particle is moving with a velocity $\bar{v}_{1}$, then according to (10) this is equivalent to an external force. Substituting (10) into (12), and setting $u=0$ and $\nabla x_{1}=0$ for a stationary gas and uniformly distributed particles, we obtain for $x_{1} \rightarrow 0$

$F_{1}=k T\left[\left(m_{1}-m_{2}\right) / m_{2}\right] \nabla \ln p=k T\left(m_{1} / m_{2}\right) \nabla \ln p$.

The last equality follows from $m_{1} / m_{2} \gg 1$. This effective force tends to move the heavy particles to the region of high pressure, which is the expected result for pressure diffusion (see reference 8, p. 244).

We could have obtained the same result without invoking Eq. (10) if we had kept the usual external force term in the general expression (12), but the above argument seems a little more straightforward.

\section{Motion in a Concentration Gradient}

Waldmann has calculated that a diffusing gas mixture exerts an effective force on a small suspended sphere. We can exhibit the same result by writing down a flux equation like (12) for the multicomponent case in a space-fixed coordinate system. This will give a result analogous to Waldmann's Eq. (7.17), but is very complicated because of the occurrence of the multicomponent diffusion coefficients. ${ }^{10}$ We therefore limit our explicit calculations here to binary gas mixtures, which from our point of view are ternary mixtures in which the suspended particles are counted as one component. For a ternary mixture in which $x_{1} \rightarrow 0$ and pressure and temperature are constant, the flux equation can be written (after considerable algebra) as

$$
\begin{aligned}
J_{1} & =n_{1} \bar{v}_{1} \\
& =-n D_{1}{ }^{*} \nabla x_{1}-n_{1} D_{1}{ }^{*}\left(1 / D_{12}-1 / D_{13}\right) D_{23} \nabla x_{2}+n_{1} u,
\end{aligned}
$$

where $D_{1}^{*}$ is the effective diffusion coefficient for a trace of 1 diffusing through a mixture of 2 and 3 , and is given by the expression

$$
1 / D_{1}^{*}=\left(x_{2} / D_{12}\right)+\left(x_{3} / D_{13}\right) .
$$

The first term of (15) gives the transport of 1 due to concentration diffusion, and the third term that due to any net flow; but the second term is new. It gives the transport of 1 due to a sort of "dragging" effect caused by the interdiffusion of 2 and 3 . Substituting for $D_{12}$ and $D_{13}$ from Eq. (8), and setting $u=0$ and $\nabla x_{1}=0$ for a stationary gas mixture and uniformly distributed particles, we obtain

$$
\bar{v}_{1}=-\left[\frac{m_{2^{\frac{1}{2}}}\left(1+\frac{4}{9} f_{2}\right)-m_{3^{\frac{1}{2}}}\left(1+\frac{4}{9} f_{3}\right)}{x_{2} m_{2^{\frac{1}{2}}}\left(1+\frac{4}{9} f_{2}\right)+x_{3} m_{3^{\frac{1}{2}}}\left(1+\frac{4}{9} f_{3}\right)}\right] D_{23} \nabla x_{2},
$$

which is equivalent to Waldmann's Eq. (7.20) with $\frac{4}{9} f$ in place of $\left(\frac{1}{8} \pi\right) a$.

The dragging effect of 2 and 3 on the trace component 1 thus causes 1 to move in the direction of diffusion of the heavier of 2 and 3 (provided $f_{2} \approx f_{3}$ ), as was pointed out by Waldmann. However, we see from (15) that the dragging effect is general for any third component present in trace concentration, since (15) is general. This effect has in fact been known for some time, ${ }^{14}$ but little attention has been paid to it.

\section{Motion in a Temperature Gradient}

We have reserved this case until last because the present elastically diffuse model gives results differing from those for Waldmann's thermally diffuse model when a temperature gradient exists. For a stationary gas and uniformly distributed particles, all at constant pressure, the flux equation for the particles when $x_{1} \rightarrow 0$ is ${ }^{10}$

$$
J_{1}=n_{1} \bar{v}_{1}=-n_{1} D_{12} \alpha_{T} \nabla \ln T .
$$

Here $\alpha_{T}$ is the thermal diffusion factor. We can calculate $\alpha_{T}$ simply as the thermal diffusion factor of a quasiLorentzian gas. By eliminating collision integrals, we can arrange the Chapman-Enskog expressions to give relations among transport properties which are independent of the particular scattering law; they depend only on the assumption that collisions are elastic., ${ }^{5,15}$ We can therefore immediately write, valid to the second approximation (and thus within the experimental error),

$$
\alpha_{T}=\frac{1}{5}\left(T \lambda_{2}{ }^{0} / p D_{12}\right)(1+\delta),
$$

${ }^{14} \mathrm{~W}$. Jost, Diffusion in Solids, Liquids, Gases (Academic Press Inc., New York, 1952), p. 431.

${ }^{15}$ S. Weissman, S. C. Saxena, and E. A. Mason, Phys. Fluids 3, 510.(1960). 
where $\lambda_{2}{ }^{0}$ is the translational thermal conductivity of the gas. This can be written in terms of the viscosity $\eta_{2}$ as

$$
\lambda_{2}^{0}=\frac{15}{4}\left(k / m_{2}\right) \eta_{2}
$$

The small quantity $\delta$ represents all the second approximation corrections lumped together. The present case is rather simple; it gives the approximate expression ${ }^{15}$

$$
\delta \approx \frac{1}{21}\left(1-d \ln \eta_{2} / d \ln T\right) \leq \frac{1}{42} .
$$

The maximum correction is only $2.4 \%$. The factor $\left(6 C_{12}{ }^{*}-5\right)$ which usually appears in (19) is unity for rigid spheres, whether specularly or diffusely reflecting. Except for the small quantity $\delta, \mathrm{Eq}$ (19) corresponds to Waldmann's Eq. (5.6), but the results differ, Waldmann's $\alpha_{T}$ being smaller by the factor $\left[1+\left(\frac{1}{8} \pi\right) a\right]$.

Substituting (19) into (18), we obtain for the drift velocity of the particles,

$$
\bar{v}_{1}=-\frac{1}{5}(1+\delta)\left(\lambda_{2}{ }^{0} / p\right) \nabla T,
$$

which differs from Waldmann's corresponding Eq. (5.2); his factor $\left[1+\left(\frac{1}{8} \pi\right) a\right]$ is missing from the denominator of (22). In terms of the effective force on the particle, we obtain by substitution of (22) into (10),

$$
F_{1}=-\frac{8}{15} r^{2}\left(2 \pi m_{2} / k T\right)^{\frac{1}{2}}(1+\delta)\left(1+\frac{4}{8} f_{2}\right) \lambda_{2}^{0} \nabla T .
$$

This should correspond to Waldmann's Eq. (4.12), except for $\delta$, but is larger by the factor $\left(1+\frac{4}{8} f\right)$.

There is thus a substantial numerical difference between the results predicted by the two models.

\section{DISCUSSION}

In summary, our results check Waldmann's in an indirect way, except when there is a temperature gradient. It is surprising that the two models agree so well up to that point, and then disagree. There is no mistake in either of the calculation procedures, however, as Professor Waldmann has proved by carrying through his calculation procedure using the elastically diffuse model. ${ }^{16} \mathrm{He}$ obtains our Eq. (23), except for $\delta$, from which (22) and (19) follow directly. We therefore have the interesting result that the general relation (19) is valid only for elastic collisions. This has interesting implications for polyatomic gases,$^{16}$ in which inelastic molecular collisions are possible. Relations among transport coefficients like (19) may not be as accurate for polyatomic gases as one might hope. Only a very few experimental measurements have yet been made to test such relations. ${ }^{15}$

It is probable that the thermally diffuse model is closer to physical reality than the elastically diffuse model, as evidenced by Schmitt's experiments. ${ }^{3}$ However, the kinetic theory calculations are much easier to carry through than the momentum transfer calculations (essentially because the difficult part of the calculation

\footnotetext{
${ }^{16} \mathrm{~L}$. Waldmann, private communication (1961).
}

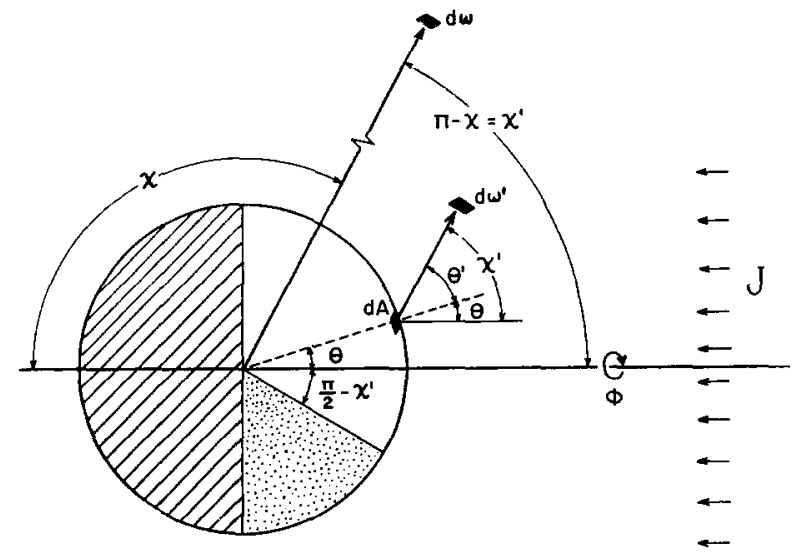

Fig. 1. Diagram for the calculation of $\sigma$ (diffuse). The shaded region of the sphere is "unilluminated" by the incoming flux $J$; the stippled region is "illuminated" by the flux but is "unseen" by a detector placed at $d \omega$.

has already been completed in the Chapman-Enskog theory). Except in the presence of temperature gradients, the elastically diffuse model should therefore be a useful approximation because it permits one to use the highly developed results of rigorous kinetic theory.

\section{ACKNOWLEDGMENT}

The authors wish to express their thanks to Professor L. Waldmann for his kindness in checking that Eq. (23) also follows by his momentum transfer procedure, and for a number of helpful comments.

\section{APPENDIX: CALCULATION OF $\sigma$ (DIFFUSE)}

We consider a flux of $J$ molecules $/ \mathrm{cm}^{2}$-sec incident on a sphere of radius $r$ (see Fig. 1). We ask how many of the incident molecules are scattered through an angle $\chi$, from the original direction of incidence into a detector which subtends a solid angle of $d \omega$. We equate this number to $J \sigma d \omega$. This is essentially the definition of $\sigma$ (which will be a function of $\chi$ ). To compute $\sigma(\chi)$ we first compute the intensity falling on an element of area of the sphere $d A$, then calculate the intensity of scattered molecules leaving $d A$ which pass into our detector $d \omega$. Finally we calculate the total intensity entering the detector, by integrating over all elements $d A$ on the surface of the sphere which can be "seen" from the detector location. This procedure is straightforward, and the notation we use is given in Fig. 1.

The intensity falling on an element of area $d A$ whose normal makes an angle $\theta$ with the direction of incoming flux is equal to $J d A \cos \theta$. On writing $d A$ in terms of $\theta$ and the azimuth angle $\phi$, this becomes

$$
J d A \cos \theta=J(r d \theta)(r \sin \theta d \phi) \cos \theta=\frac{1}{2} J r^{2} \sin 2 \theta d \theta d \phi .
$$

For diffuse reflection, the fraction of molecules leaving $d A$ at an angle $\theta^{\prime}$ from the normal to the surface is proportional to $\cos \theta^{\prime}$ (the cosine law for diffuse reflection $\left.^{6}\right)$. Therefore the fraction leaving $d A$ at $\theta^{\prime}$ and 
passing through solid angle $d \omega^{\prime}=\sin \theta^{\prime} d \theta^{\prime} d \phi\left(d \omega^{\prime}\right.$ is not the same as $d \omega$; see Fig. 1) is

$$
\frac{1}{2} \frac{\cos \theta^{\prime} d \omega^{\prime}}{\int \cos \theta^{\prime} d \omega^{\prime}}=\cos \theta^{\prime}\left(\frac{d \omega^{\prime}}{d \phi}\right) .
$$

The latter expression is obtained by carrying out the integration in the normalization factor of the denominator. The factor $\frac{1}{2}$ appears in (A2) because only half the intensity leaving at $\theta^{\prime}$ passes through our selected element $d \omega^{\prime}$; the other half passes through a different element located symmetrically on the other side of the normal. Combining (A1) and (A2), we find that the intensity leaving $d A$ at $\theta^{\prime}$ and passing through $d \omega^{\prime}$ is

$$
\frac{1}{2} J r^{2} \sin 2 \theta \cos \theta^{\prime} d \theta d \omega^{\prime} .
$$

But we want the intensity leaving $d A$ at $\theta^{\prime}$ and passing through $d \omega=\sin \chi^{\prime} d \chi^{\prime} d \phi$, where $\chi^{\prime}=\pi-\chi$. Now $d \omega \neq d \omega^{\prime}$, but $d \chi^{\prime}=d \theta^{\prime}$. Thus the relation between $d \omega$ and $d \omega^{\prime}$ is

$$
d \omega^{\prime}=\left(\sin \theta^{\prime} / \sin \chi^{\prime}\right) d \omega .
$$

Substituting (A4) into (A3), we find that the intensity leaving $d A$ at $\theta^{\prime}$ and passing through $d \omega$ is

$\frac{1}{2} J r^{2} \sin 2 \theta \cos \theta^{\prime} d \theta\left(\sin \theta^{\prime} / \sin \chi^{\prime}\right) d \omega$

$$
=\left(J r^{2} d \omega / 4 \sin \chi^{\prime}\right) \sin 2 \theta \sin 2 \theta^{\prime} d \theta .
$$

To complete the calculation we need only to integrate (A5) over the available range of $\theta$ which is "seen" by the detector at $d \omega$, and equate the result to $J \sigma(\chi) d \omega$. However, we must first express $\theta^{\prime}$ as a function of $\theta$ and $\chi$. This leads us to divide up the available range of $\theta$ into several regions. We consider first the case of $\chi^{\prime}$ between 0 and $\frac{1}{2} \pi$ (this is the one explicitly shown in Fig. 1); i.e., $\chi$ is between $\frac{1}{2} \pi$ and $\pi$; we distinguish three regions:

Region 1: From $\theta=0$ to $\theta=\frac{1}{2} \pi-\chi^{\prime}$. In this region we have $\theta^{\prime}=\theta+\chi^{\prime}$, and the integration of (A5) over $\theta$ yields the following contribution to $\sigma(\chi)$ :

$$
\sigma_{1}\left(\chi^{\prime}\right)=\left(\frac{1}{18} r^{2}\right)\left(\sin \chi^{\prime}\right)^{-1}\left[\left(\pi-2 \chi^{\prime}\right) \cos 2 \chi^{\prime}+\sin 2 \chi^{\prime}\right] .
$$

Region 2: From $\theta=0$ to $\theta=\chi^{\prime}$; here we have $\theta^{\prime}=\chi^{\prime}-\theta$, and the integration yields

$$
\sigma_{2}\left(\chi^{\prime}\right)=\left(\frac{1}{16} r^{2}\right)\left(\sin \chi^{\prime}\right)^{-1}\left[\sin 2 \chi^{\prime}-2 \chi^{\prime} \cos 2 \chi^{\prime}\right] \text {. }
$$

Region 3: From $\theta=\chi^{\prime}$ to $\theta=\frac{1}{2} \pi$; here we have $\theta^{\prime}=$ $\theta-\chi^{\prime}$, and the integration yields

$$
\sigma_{3}\left(\chi^{\prime}\right)=\sigma_{1}\left(\chi^{\prime}\right) .
$$

Adding together (A6), (A7), and (A8), and converting from $\chi^{\prime}$ to $\chi=\pi-\chi^{\prime}$, we obtain the final result given by Eq. (4b).

The case of $\chi^{\prime}$ between $\frac{1}{2} \pi$ and $\pi$ (i.e., $\chi$ between 0 and $\left.\frac{1}{2} \pi\right)$ is simpler. Only one region need be considered, extending from $\theta=\chi^{\prime}-\frac{1}{2} \pi$ to $\theta=\frac{1}{2} \pi$. Here we have $\theta^{\prime}=\chi^{\prime}-\theta$, and the integration of (A5) over $\theta$ yields

$$
\sigma\left(\chi^{\prime}\right)=\left(\frac{1}{16} r^{2}\right)\left(\sin \chi^{\prime}\right)^{-1}\left[-2\left(\pi-\chi^{\prime}\right) \cos 2 \chi^{\prime}-\sin 2 \chi^{\prime}\right] \text {. }
$$

This yields the final result given by Eq. (4a) on converting from $\chi^{\prime}$ to $\chi$. 